\title{
Derivation of the Bi-axial Bending, Compression and Shear Strengths of Timber Beams
}

\author{
T. A. C. M. Van Der Put ${ }^{*}$ \\ Faculty of Civil Engineering and Geosciences, Timber Structures and wood technology, TU Delft, P.O. Box 5048, 2600 \\ GA Delft The Netherlands
}

\begin{abstract}
The derivation is given of the combined bi-axial bending, compression and shear strength of timber beams. As for other materials the elastic-full plastic limit design approach applies, which is known to precisely explain and predict uniaxial bending strength behaviour.

The derivation is based on choosing the location of the neutral line. This provides the stress distribution in the beam cross section in the ultimate state for that case, making it possible to calculate the associated ultimate bending moments in both main directions and ultimate normal- and shear force. The derived general equations are simplified to possible elementary design equations, applicable for building regulation.
\end{abstract}

Keywords: Timber beams, limit analysis, bi-axial bending-compression-shear strength.

\section{INTRODUCTION}

As known, timber beams behaves quasi isotropic for the loading case of bending, compression with shear up to the ultimate state [1] and the common beam theory can be applied. The fictive bending strength $f_{m}$, based on the linearized bending stress in the failure state, given in [2], only applies for rectangular cross-sections and for the most elementary loading case. For combined loading cases and to explain measurements, the elastic-full plastic diagram has to be used as shown e.g. in [3], where the derivation is given of the uniaxial bending, compression and shear strength of timber beams. For profiles this elastic-plastic approach has to be applied to obtain the necessary profile factors on the fictive linear bending strength $f_{m}$. The elastic-full plastic approach is the basis for limit design and applies for all materials and it is extensively shown also for other materials as steel and concrete [4] to be sufficient for the real strength prediction. For wood this necessary design method was already generally known and widely applied since 1930 (see [5]). Although necessary as basis for stability design and for the prescribed calculable reliability, bi-axial bending strength combined with compression and shear loading is never determined mathematically before and therefore is given here to correct this omission.

The elastic-plastic stress diagram, with a negligible plastic range for tension, applied in the figures below, represents an admissible equilibrium system, satisfying equilibrium and boundary conditions, violating nowhere the yield criterion, and thus is lower bound solution. The highest lower bound solution is equal to the real strength and this is reached in this

*Address correspondence to this author at the Faculty of Civil Engineering and Geosciences, Timber Structures and wood technology, TU Delft, P.O. Box 5048, 2600 GA Delft The Netherlands; Tel: +31152851980;

Fax: +31208509239; E-mail: vanderp@xs4all.nl case when the neutral line is a straight line and when unlimited flow in pure compression is possible, thus when the shear stress is carried in the elastic part of the cross section.

Thus as confirmed in [3], the uniaxial ultimate combined bending-compression strength is determined by the ultimate tensile stress $f_{t}$ and by unlimited "flow" in compression at the flow compression stress $f_{c}$. Bending failure thus always is an ultimate tension failure at $f_{t}$. This therefore is the starting point for the derivations in Section 2 and is an improvement with respect to the old model, applied in [6], which was based on a limited ultimate compression strain and therefore did not fit precisely to the data.

The derivations of Section 2 are in principle based on choosing the location of the neutral line and calculate the associated ultimate bending moments and normal and shear forces. There are three cases to regard for the location of the neutral line. The neutral line may go through two opposite planes of the cross section as given in Fig. (1), or the neutral line goes through two adjacent planes, at the tension side or at the compression side as given by respectively Fig. ( 6 and 7). Mathematically simpler is not to choose the location of the neutral line but of the parallel border line of the full plastic compression area of the cross section as done in the following.

\section{BI-AXIAL BENDING STRENGTH CASES}

2.1. Dominating Bending in the Stiff Direction $(Y \geq b$; $Z \leq h$ in Fig. 1)

The ultimate state of the determining cross-section of a beam loaded under biaxial bending is given in Figs. (1, $\mathbf{6}$ and 7). The line EF in Fig. (1) is the boundary of the full plastic, ultimate compression strength area of the cross-section of a beam. In Fig. (1) an equilibrium state is given of a beam with dimension $b$ and $h$, loaded in "double" bending. For the analysis, the bending stresses of the ultimate state are re- 


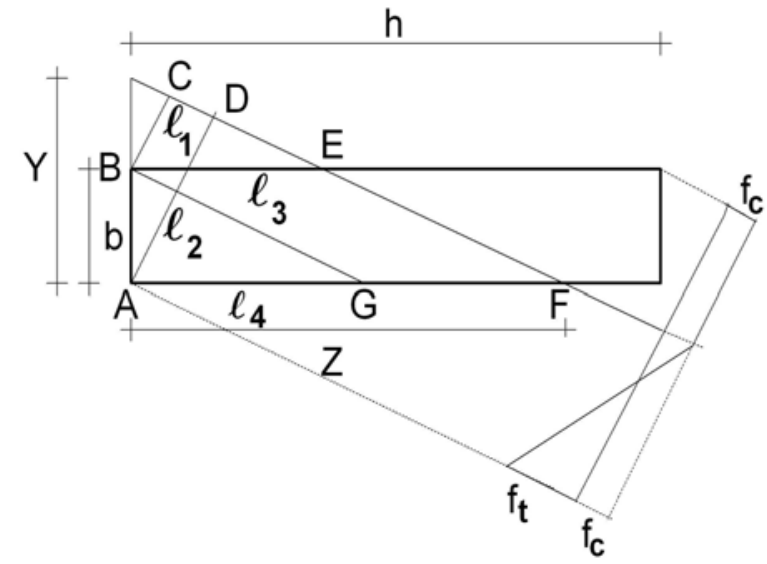

Fig. (1). Compression with bi-axial bending.

garded to be a superposition of compression force $N_{u}=f_{c} b h$ of the uniform compression stress $f_{c}$ over the entire cross-section and a tension force by the linear increasing tensile stresses in the plane ABEF with a maximal tensile stress $f_{t}+f_{c}$ in point $\mathrm{A}$.

In Fig. (1) is: $\ell_{1}=$ line $\mathrm{BC}, \ell_{2}=$ line $\mathrm{AD}, \ell_{3}=$ line $\mathrm{BE}, \ell_{4}$ $=$ line $\mathrm{AG}$. Then:

$\frac{\ell_{1}}{\ell_{2}}=\frac{Y-b}{Y}=1-\frac{b}{Y}, \frac{\ell_{3}}{Z}=\frac{Y-b}{Y}=1-\frac{b}{Y}, \frac{\ell_{4}}{Z}=\frac{b}{Y}$

\subsubsection{Normal Forces}

The tensile stress at point B is, using eq.(1):

$\sigma_{B}=\frac{\ell_{1}}{\ell_{2}}\left(f_{t}+f_{c}\right)=\left(1-\frac{b}{Y}\right) \cdot\left(f_{t}+f_{c}\right)$

The total tensile force $T$ consists of the sum of stresses within the stress pyramid of $T_{1}$ in the center of gravity of this stress pyramid above plane ABG with maximal stress at A of $f_{t}+f_{c}-\sigma_{B}$ and the part $T_{2}$ above plane ABG, below the stress pyramid $T_{1}$ with constant stress $\sigma_{B}$ and finally of $T_{3}$ above plane BEFG, with a linear increasing stress to $\sigma_{B}$ at points B and G. Then is, using eq.(1) and (2):

$T_{1}=\frac{f_{t}+f_{c}-\sigma_{B}}{3} \cdot \frac{b \ell_{4}}{2}=\left(f_{t}+f_{c}\right) \cdot \frac{b^{3} Z}{6 Y^{2}}$,

$T_{2}=\sigma_{B} \cdot \frac{b \ell_{4}}{2}=\left(f_{t}+f_{c}\right) \cdot \frac{b^{2} Z}{2 Y} \cdot\left(1-\frac{b}{Y}\right)$,

$T_{3}=\sigma_{B} \cdot \frac{b \ell_{3}}{2}=\left(f_{t}+f_{c}\right) \cdot \frac{b Z}{2} \cdot\left(1-\frac{b}{Y}\right)^{2}$,

$T=T_{1}+T_{2}+T_{3}=\left(f_{t}+f_{c}\right) \cdot\left[\frac{b^{3} Z}{6 Y^{2}}+\frac{b^{2} Z}{2 Y} \cdot\left(1-\frac{b}{Y}\right)+\frac{b Z}{2} \cdot\left(1-\frac{b}{Y}\right)^{2}\right]$

Thus, the ultimate value of $T$ is:

$T_{u}=\left(f_{t}+f_{c}\right) \frac{b Z}{2}\left(1-\frac{b}{Y}+\frac{b^{2}}{3 Y^{2}}\right)$

The ultimate normal force $N_{u}$, with (for convenience) a positive sign for compression, is:
$N_{u}=f_{c} b h-T=f_{c} b h-\left(f_{t}+f_{c}\right) \frac{b Z}{2}\left(1-\frac{b}{Y}+\frac{b^{2}}{3 Y^{2}}\right)=f_{c} b h\left[1-(s+1) \frac{Z}{2 h}\left(1-\frac{b}{Y}+\frac{b^{2}}{3 Y^{2}}\right)\right](7)$

where $s=f_{t} / f_{c}$. Thus, with the maximal possible value of $N_{u}$ is, with $N_{u \cdot m}=f_{c} b h$ :

$(s+1) \frac{Z}{2 h}\left(1-\frac{b}{Y}+\frac{b^{2}}{3 Y^{2}}\right)=1-\frac{N_{u}}{N_{u, m}}$

For uniaxial bending in the stiff direction, $Y \rightarrow \infty$ (or: $b / Y \rightarrow 0$ ), this equation agrees with Eq. (2) of [3], where it was shown that the theory precisely fits the data.

Because of the boundary conditions of the equations of Section 2.1: $Y \geq b ; Z \leq h$ is: $1 \geq N_{u} / N_{u, m} \geq-(s-1) / 2$, (9) with a tension limit (negative sign) when $s>1$, for the equations of Section 2.1.

\subsubsection{Bending Moments}

The bending moment by $T=T_{1}+T_{2}+T_{3}$, according to eq. (3) to (6), with respect to the resultant compression force $N_{u, m}=f_{c} b h$, thus with respect to the center of the crosssection of the beam, is: in the stiff direction:

$M_{y}=T_{1}\left(h / 2-\ell_{4} / 4\right)+T_{2}\left(h / 2-\ell_{4} / 3\right)+T_{3}\left(h / 2-\ell_{4} / 2-\ell_{3} / 3\right)=$

$=T \cdot h / 2-T_{1} \ell_{4} / 4-T_{2} \ell_{4} / 3-T_{3} \cdot\left(\ell_{4} / 2+\ell_{3} / 3\right)$

and in the weak direction:

$M_{z}=T_{1}(b / 2-b / 4)+T_{2}(b / 2-b / 3)+0=T_{1} b / 4+T_{2} b / 6$

Thus, using eqs.(1 to 6$)$ :

$M_{y}=\left(f_{t}+f_{c}\right) \frac{b Z h}{4}\left(1-\frac{b}{Y}+\frac{b^{2}}{3 Y^{2}}\right)-\left(f_{t}+f_{c}\right) \frac{b Z^{2}}{12}\left(2-3 \frac{b}{Y}+2 \frac{b^{2}}{Y^{2}}-\frac{b^{3}}{2 Y^{3}}\right)$

or, by substitution of $Z$ according to Eq.(8), this equation becomes:

$M_{y}=f_{c} \frac{b h^{2}}{6}\left(1-\frac{N_{u}}{N_{u, m}}\right)\left[3-\frac{1-N_{u} / N_{u, m}}{s+1} \cdot \frac{4-6 b / Y+4 b^{2} / Y^{2}-b^{3} / Y^{3}}{\left(1-b / Y+b^{2} /\left(3 Y^{2}\right)\right)^{2}}\right]$

$M_{z}=\left(f_{c}+f_{t}\right) \frac{b^{3} Z}{12 Y}\left(1-\frac{b}{2 Y}\right)$

By substitution of $Z$ according to Eq.(8), Eq.(14) becomes:

$M_{z}=f_{c} \frac{h b^{2}}{6} \cdot \frac{b / Y(1-b / 2 Y)}{1-b / Y+b^{2} / 3 Y^{2}} \cdot\left(1-\frac{N_{u}}{N_{u, m}}\right)$

For $Y \rightarrow \infty, M_{z}=0$ as follows from Eq. (14) or (15) and thus uniaxial bending occurs and Eq. (8) then becomes: $(s+1) Z /(2 h)=1-N_{u} / N_{u, m}$.

When this is substituted in Eq. (12) for $Y \rightarrow \infty$, the uniaxial bending strength becomes:

$M_{y, \infty}=\left(f_{t}+f_{c}\right) \frac{b Z h}{4}-\left(f_{t}+f_{c}\right) \frac{b Z^{2}}{6}=f_{c} \frac{b h^{2}}{6} \cdot \frac{-1+3 s+4 N_{u} / N_{u, m}}{s+1} \cdot\left(1-\frac{N_{u}}{N_{u, m}}\right)(16)$

as found before in [3] based on the data of [6]. 


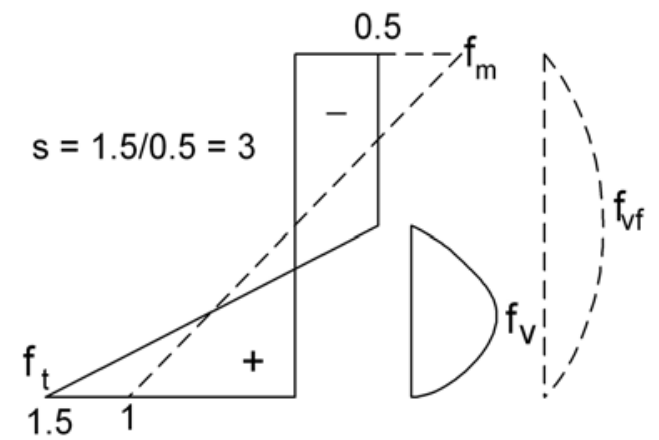

Fig. (2). Bending and shear stress.

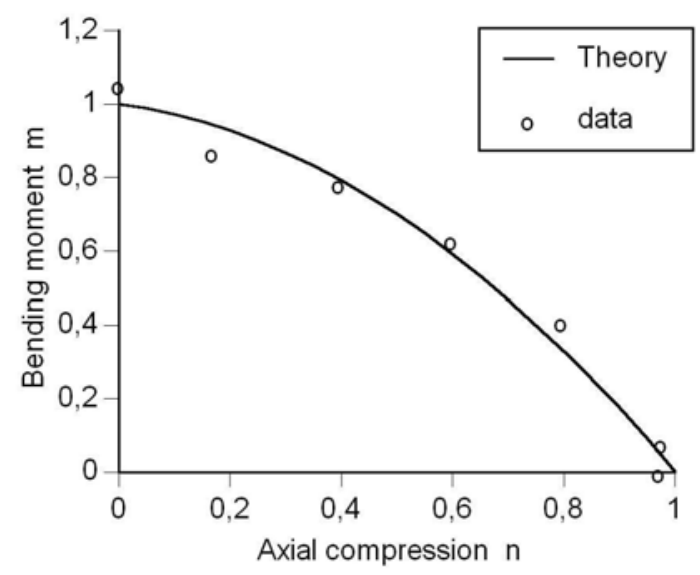

Fig. (3). $s=2-95$ th percentile of the bending compression strength.

The fictive linear elastic design bending stress, applied in the Building Codes, thus is:

$\sigma_{m}=\frac{6 M_{y, \infty}}{b h^{2}}=f_{c} \cdot \frac{-1+3 s+4 N_{u} / N_{u, m}}{s+1} \cdot\left(1-\frac{N_{u}}{N_{u, m}}\right)$

which is equal to the uniaxial bending strength $f_{m}$ when $N=0$, Thus when:

$f_{m}=\frac{6 M_{y, \infty}}{b h^{2}}=f_{c} \frac{3 s-1}{s+1}$

given in Fig. (2). In [3], the value of $s=1.3$ was found for the mean strength, while $s=2$ for the 95th percentile and $s=0.77$ for the 5 th percentile of the uniaxial combined bending - compression strength, given in Figs. (3, 4 and 5) of [3], where $m=6 M_{u} / f_{m} b h^{2}$ and $n=N_{u} / f_{c} b h$. These values are based on the data of [6] and apply for standard climate conditions. Important is, that these values of $s$ are independent of the load-combination, showing that there is no volume effect due to tensile stress distribution but only for volume alone. This is explained by a decrease of quality with volume increase. This also explains why by not brittle compression failure a volume effect is possible (as reported in literature).

According to the boundary conditions, the equations of Section 2.1.2 apply for:

$f_{c} b h^{2}(s+1) / 24 \leq M_{y} \leq f_{c} b h^{2}(s+1) / 12$

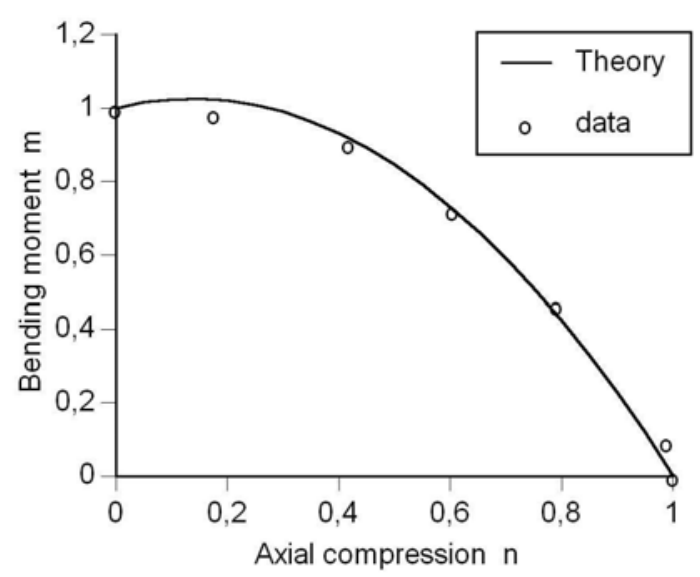

Fig. (4). $s=1.3$ - 50th strength percentile.

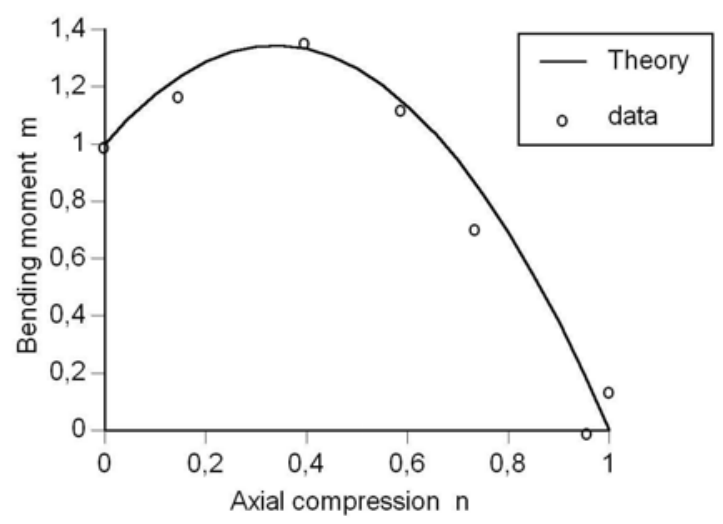

Fig. (5). $s=0.77$ - 5 th strength percentile.

and

$0 \leq M_{z} \leq f_{c} b h^{2}(s+1) / 24$

\subsubsection{Shear Force}

The total ultimate resulting shear force $V_{u}\left(=\sqrt{V_{x}^{2}+V_{y}^{2}}\right)$ in the elastic region of the cross section is:

$V_{u}=\frac{2}{3} f_{v}\left(b \ell_{3}+\frac{b\left(Z-\ell_{3}\right)}{2}\right)=\frac{2}{3} f_{v} b Z\left(1-\frac{b}{2 Y}\right)$

based on the parabolic shear stress distribution in the elastic region. The possible range of $V_{u}$ is: $0 \leq V_{u} \leq 2 f_{v} b h / 3$ for all cases of Section 2.1.

Substitution of $Z$ of Eq.(8) into Eq.(20) gives:

$V_{u}=\frac{2}{3} f_{v} b h\left(1-\frac{b}{2 Y}\right) \cdot\left(\frac{1-N_{u} / N_{u, m}}{1-b / Y+b^{2} /\left(3 Y^{2}\right)}\right) \cdot \frac{2}{s+1}$

or with uniaxial $V_{0, \infty}$ according to Eq.(23):

$\frac{V_{u}}{V_{0, \infty}}=\left(1-\frac{b}{2 Y}\right)\left(\frac{1-N_{u} / N_{u, m}}{1-b / Y+b^{2} /\left(3 Y^{2}\right)}\right)$

The shear strength $V_{0, \infty}$ is determined at ultimate uniaxial bending, for $N=0$ and $Y \rightarrow \infty$ and is given for design as a fictive linear elastic parabolic stress distribution over the total depth $h$. according to Fig. (2). Thus 


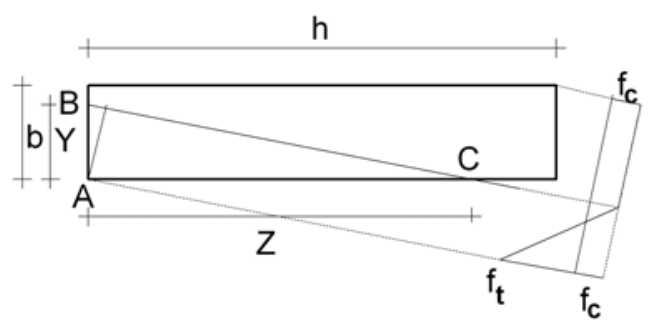

Fig. (6). Dominating compression with bi-axial bending for $Z \leq h$ and $Y \leq b$.

$V_{0, \infty}=\frac{2}{3} f_{v} b h \frac{2}{s+1}=\frac{2}{3} f_{v, f} b h$

For $Y \rightarrow \infty$, Eq. (22) represents the uniaxial loading case (see [3]) giving:

$\frac{V_{u, \infty}}{V_{0, \infty}}=1-\frac{N_{u}}{N_{u, m}}$

\subsection{Dominating Bending in the Weak Direction ( $Z \geq h$} and $\boldsymbol{Y} \leq \boldsymbol{b}$ )

All equations thus far apply for the case of Fig. (1), thus for $Z \leq h$ and $Y \geq b$. For $Z \geq h$ and $Y \leq b$, the same equations of Section 2.1 apply with interchange of $z$ and $y ; Z$ and $Y, b$ and $h$ as follows by eqs. (25 to 27):

$$
\begin{aligned}
& M_{z}=f_{c} \frac{h b^{2}}{6}\left(1-\frac{N_{u}}{N_{u, m}}\right)\left[3-\frac{1-N_{u} / N_{u, m}}{s+1} \cdot \frac{4-6 h / Z+4 h^{2} / Z^{2}-h^{3} / Z^{3}}{\left(1-h / Z+h^{2} /\left(3 Z^{2}\right)\right)^{2}}\right] \\
& M_{y}=f_{c} \frac{b h^{2}}{6} \frac{1-h / 2 Z}{-1+Z / h+h / 3 Z}\left(1-\frac{N_{u}}{N_{u, m}}\right) \\
& \frac{V_{u}}{V_{0, \infty}}=\left(\frac{1-h / 2 Z}{1-h / Z+h^{2} /\left(3 Z^{2}\right)}\right) \cdot\left(1-\frac{N_{u}}{N_{u, m}}\right)
\end{aligned}
$$

Again one component of the biaxial moment shows the linear relation with $\left(1-N / N_{u, m}\right)$. When Eq.(27) is determining, also curve Eq.(25) is cut off to this relation (see Section 4).

\subsection{Dominating loading in compression $(Y \leq b$ and $Z \leq h$ in Fig. 6)}

For dominating compression, the condition $Y \leq b$ and $Z \leq h$ may apply according to Fig. (6).

\subsubsection{Normal Forces}

$N_{u}=f_{c} b h-\frac{1}{2} Z Y \cdot \frac{1}{3}\left(f_{t}+f_{c}\right)=f_{c} b h\left(1-\frac{s+1}{6} \cdot \frac{Y Z}{b h}\right)$ or:

$\frac{s+1}{6} \cdot \frac{Y Z}{b h}=1-\frac{N_{u}}{N_{u, m}}$

According to the boundary conditions is

$$
((5-s) / 6) \leq N_{u} / N_{u, m} \leq 1 \text {. }
$$

\subsubsection{Shear Force}

For the ultimate shear force applies:

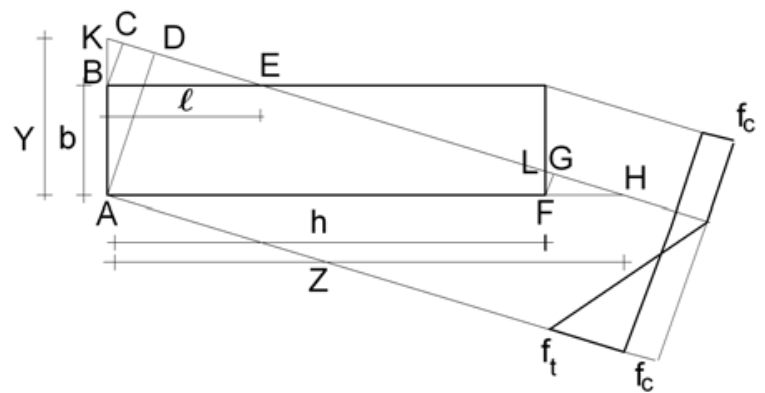

Fig. (7). Dominating tension with bi-axial bending for $Z \geq h$ and.

$V_{u}=\frac{2}{3} f_{v} \frac{Y Z}{2}=f_{v} \frac{Y Z}{3}=f_{v} b h \frac{2}{s+1}\left(1-\frac{N_{u}}{N_{u, m}}\right)$

Due to the boundary conditions $Z \leq h$ and $Y \leq b$, is $V_{u}$ here maximal: $V_{u, m}=f_{v} \frac{b h}{3}$.

From Eq. (30) follows, when shear strength is determining:

$V_{u}=f_{v} \frac{Y Z}{3}=f_{v} b h\left(1-\frac{N_{u}}{N_{u, m}}\right) \frac{2}{s+1} \leq f_{v} \frac{b h}{3}$

because according to Eq. (29): $N_{u} / N_{u, m} \geq((5-s) / 6)$

\subsubsection{Bending Moments}

As before, the resultant force of the tensile stress pyramid times the distance to the resultant compression force in the center of the cross section determines the bending moment.

For bending applies, using Eq. (28):

$$
\begin{aligned}
& M_{y}=\frac{1}{3}\left(f_{c}+f_{t}\right) \frac{Y Z}{2}\left(\frac{h}{2}-\frac{Z}{4}\right) \leq f_{c} b h \frac{h}{4}\left(1-\frac{N_{u}}{N_{u, m}}\right) \\
& M_{z}=\frac{1}{3}\left(f_{c}+f_{t}\right) \frac{Y Z}{2}\left(\frac{b}{2}-\frac{Y}{4}\right) \leq f_{c} b h \frac{b}{4}\left(1-\frac{N_{u}}{N_{u, m}}\right)
\end{aligned}
$$

Knowing $M_{y}, N$ and $M_{z}, \mathrm{Y}$ and $\mathrm{Z}$ are known and the found product $Y Z$ should be smaller for bending failure than the value of $Y Z$ for shear failure according to Eq.(30), thus

$$
(Y Z)_{\text {bending }} \leq\left(3 V_{u} / f_{v}\right)
$$

There is no advantage to first eliminate $h / Z$ and to end with an expression in $b / Y$ as done before for the cases of Sections 2.1 and 2.2.

\subsection{Dominating Loading in Tension}

For dominating tension, the condition $Y \geq b$ and $Z \geq h$ may apply according to Fig. (7).

In Fig. (7) is:

$\frac{\ell}{Z}=\frac{K B}{K A}=\frac{Y-b}{Y} \frac{L F}{Y}=\frac{Z-h}{Z}$

In the limit case for the here applied equations, when $\ell=$ $h$ and line $\mathrm{LF}=\mathrm{b}$, is:

$\frac{b}{Y}+\frac{h}{Z}=1$ 


\subsubsection{Normal Forces}

The tension force $T$ follows from the content of the tension pyramid AHKA with height $f_{t}+f_{c}$ minus the contents of pyramids BEKB and FHLF with heights $\sigma_{B}$ and $\sigma_{F}$ with:

$\sigma_{F}=\frac{G F}{A D}\left(f_{t}+f_{c}\right)=\frac{H F}{H A}\left(f_{t}+f_{c}\right)=\frac{Z-h}{Z}\left(f_{t}+f_{c}\right)$

$\sigma_{B}=\frac{B C}{A D}\left(f_{t}+f_{c}\right)=\frac{K B}{K A}\left(f_{t}+f_{c}\right)=\frac{Y-h}{Y}\left(f_{t}+f_{c}\right)$

Thus the total tension force is:

$T=T_{A}-T_{F}-T_{B}$

with:

$T_{A}=\frac{f_{t}+f_{c}}{3} \frac{Y Z}{2}$

$T_{F}=\frac{\sigma_{F}}{3} \frac{L F \cdot F H}{2}=\frac{f_{t}+f_{c}}{6}\left(1-\frac{h}{Z}\right)\left(1-\frac{h}{Z}\right) Y(Z-h)=\frac{f_{t}+f_{c}}{6} Y Z\left(1-\frac{h}{Z}\right)^{3}$

$=T_{A}\left(1-\frac{h}{Z}\right)^{3}$

In the same way is:

$T_{B}=\frac{f_{t}+f_{c}}{6} Y Z\left(1-\frac{b}{Y}\right)^{3}=T_{A}\left(1-\frac{b}{Y}\right)^{3}$

Thus:

$T=T_{A}\left(1-\left(1-\frac{b}{Y}\right)^{3}-\left(1-\frac{h}{Z}\right)^{3}\right)$

The applied ultimate normal compression force is:

$N_{u}=f_{c} b h-T_{u}=f_{c} b h\left(1-\frac{s+1}{6} \frac{Y Z}{b h}\left(1-\left(1-\frac{b}{Y}\right)^{3}-\left(1-\frac{h}{Z}\right)^{3}\right)\right)$

To replace $Y Z$ in other equations, this can be written with $N_{u, m}=f_{c} b h$ :

$\frac{s+1}{6} \frac{Y Z}{b h}\left(1-\left(1-\frac{b}{Y}\right)^{3}-\left(1-\frac{h}{Z}\right)^{3}\right)=1-\frac{N_{u}}{N_{u, m}}$.

In the limit case of Eq.(45) is for: $h=Z, Y \rightarrow \infty$ (or $b / Y \rightarrow 0)$ :

$\frac{s+1}{6} \frac{Y}{b}\left(1-\left(1-\frac{b}{Y}\right)^{3}\right)=\frac{s+1}{6} \frac{Y}{b}\left(1-\left(1-3 \frac{b}{Y}+3\left(\frac{b}{Y}\right)^{2}-\left(\frac{b}{Y}\right)^{3}\right)\right) \approx \frac{s+1}{2}=1-\frac{N_{u}}{N_{u, m}}(46)$

The same applies for $b=Y, Z \rightarrow \infty$.

\subsubsection{Shear Force}

The ultimate shear force is:

$$
V_{u}=\frac{2}{3} f_{v}\left(b h-\frac{(b-F L)(h-\ell)}{2}\right)=\frac{2}{3} f_{v} b h\left(1-\frac{\left(b-\frac{Z-h}{Z} Y\right)\left(h-\frac{Y-b}{Y} Z\right)}{2 b h}\right)=
$$

$$
\begin{aligned}
& =\frac{2}{3} f_{v} b h\left(1-\frac{1}{2}\left(1-\left(1-\frac{h}{Z}\right) \frac{Y}{b}-\left(1-\frac{b}{Y}\right) \frac{Z}{h}+\left(1-\frac{h}{Z}\right)\left(1-\frac{b}{Y}\right) \frac{Z Y}{b h}\right)\right)= \\
& =\frac{2}{3} f_{v} \frac{b h}{2}\left(\frac{2 Y}{b}+\frac{2 Z}{h}-\frac{h Y}{b Z}-\frac{b Z}{h Y}-\frac{Z Y}{b h}\right)
\end{aligned}
$$

In the limit case is for: $(h=Z, Y \rightarrow \infty)$ or for $(b=Y$, $Z \rightarrow \infty): V_{u}=2 f_{v} b h / 3$.

\subsubsection{Bending Moments}

The ultimate bending moment is:

$$
\begin{aligned}
& M_{y}=T_{A}\left(\frac{h}{2}-\frac{Z}{4}\right)-T_{B}\left(\frac{h}{2}-\frac{\ell}{4}\right)-T_{F}\left(\frac{h}{2}-\left(h+\frac{Z-h}{4}\right)\right)= \\
& T \frac{h}{2}-T_{A} \frac{Z}{4}+T_{B} \frac{\ell}{4}+T_{F}\left(h+\frac{Z-h}{4}\right)= \\
& =T_{A}\left(1-\left(1-\frac{b}{Y}\right)^{3}-\left(1-\frac{h}{Z}\right)^{3}\right) \frac{h}{2}-T_{A} \frac{Z}{4}+T_{A}\left(1-\frac{b}{Y}\right)^{3} \frac{\ell}{4}+T_{A}\left(1-\frac{h}{Z}\right)^{3}\left(h+\frac{Z-h}{4}\right)= \\
& =T_{A}\left(\frac{h}{2}-\left(1-\frac{b}{Y}\right)^{3} \frac{h}{2}-\left(1-\frac{h}{Z}\right)^{3} \frac{h}{2}-\frac{Z}{4}+\frac{Z}{4}\left(1-\frac{b}{Y}\right)^{4}+\left(1-\frac{h}{Z}\right)^{3}\left(h+\frac{Z-h}{4}\right)\right)= \\
& =T_{A} \frac{h}{2}\left(1-\left(1-\frac{b}{Y}\right)^{3}-\left(1-\frac{h}{Z}\right)^{3}-\frac{Z}{2 h}+\frac{Z}{2 h}\left(1-\frac{b}{Y}\right)^{4}+\left(1-\frac{h}{Z}\right)^{3}\left(\frac{3}{2}+\frac{Z}{2 h}\right)\right)(48
\end{aligned}
$$

Thus:

$M_{y}=f_{c} \frac{(s+1)}{2} \cdot \frac{Y Z h}{6} \cdot\left(1-\left(1-\frac{b}{Y}\right)^{3}-\left(1-\frac{h}{Z}\right)^{3}-\frac{Z}{2 h}+\frac{Z}{2 h}\left(1-\frac{b}{Y}\right)^{4}+\left(1-\frac{h}{Z}\right)^{3}\left(\frac{3}{2}+\frac{Z}{2 h}\right)\right)$

For the limit case in accordance with Eq.(36): $h=Z$, $Y \rightarrow \infty$ applies for $M_{y}$, giving:

$M_{y}=f_{c} \frac{s+1}{2} \frac{b h^{2}}{6}$.

The limit case: $b=Y, Z \rightarrow \infty$ applies to $M_{Z}$ leading to

$M_{z}=f_{c} \frac{s+1}{2} \cdot \frac{h b^{2}}{6}$

In general is $M_{z}$ :

$M_{z}=T_{A}\left(\frac{b}{2}-\frac{Y}{4}\right)-T_{B}\left(\frac{b}{2}-\left(b+\frac{Y-b}{4}\right)\right)-T_{F}\left(\frac{b}{2}-\frac{Y}{4}\left(1-\frac{h}{Z}\right)\right)=$

$=T \frac{b}{2}-T_{A} \frac{Y}{4}+T_{B} \frac{3 b-Y}{4}+T_{F}\left(1-\frac{h}{Z}\right) \frac{Y}{4}=$

$=T_{A}\left(\left(1-\left(1-\frac{b}{Y}\right)^{3}-\left(1-\frac{h}{Z}\right)^{3}\right) \frac{b}{2}-\frac{Y}{4}+\left(1-\frac{b}{Y}\right)^{3} \frac{3 b+Y}{4}+\left(1-\frac{h}{Z}\right)^{4} \frac{Y}{4}\right)$.

Thus:

$M_{z}=f_{c} \frac{s+1}{12} Y Z b\left(\left(1-\left(1-\frac{b}{Y}\right)^{3}-\left(1-\frac{h}{Z}\right)^{3}\right)-\frac{Y}{2 b}+\left(1-\frac{b}{Y}\right)^{3}\left(\frac{3}{2}+\frac{Y}{2 b}\right)+\left(1-\frac{h}{Z}\right)^{4} \frac{Y}{2 b}\right)(52)$

\subsubsection{High Tensional Loading}

At higher tensile loading the behaviour is linear elastic and determining for failure is the maximal tension stress leading to: 
$f_{t}=\frac{6 M_{y}}{b h^{2}}+\frac{6 M_{z}}{h b^{2}}+\frac{N_{u}}{b h}$ or: $1=\frac{M_{y}}{M_{y, u}}+\frac{M_{z}}{M_{z, u}}+\frac{N_{u}}{N_{t, u}}$

In this equation tension has the positive sign.

\section{SIMPLIFICATION FOR PRACTICAL DESIGN}

For practical design at dominating bending, the expressions in variables $b / Y$ and $h / Z$ in the equations can be simplified without loss of accuracy.

\subsection{Dominating Compression}

For dominating compression, $(Z \leq h$ and $Y \leq b)$, Eqs. (28) to (34) are simple enough as design equations.

\subsection{Dominating Bending in the Stiff Direction}

Eq.(13) for dominating bending in the stiff direction $(Z \leq h$ and $Y \geq b)$ is:

$M_{y}=f_{c} \frac{b h^{2}}{6}\left(1-\frac{N_{u}}{N_{u, m}}\right)\left[3-\frac{1-N_{u} / N_{u, m}}{s+1} \cdot \Phi_{1}\left(\frac{b}{Y}\right)\right]$

with: $\Phi_{1}(b / Y)=\frac{4-6 b / Y+4 b^{2} / Y^{2}-b^{3} / Y^{3}}{\left(1-b / Y+b^{2} /\left(3 Y^{2}\right)\right)^{2}}$.

In the applying range ( $Z \leq h$ and $b / Y$ between 0 to 1 ), $\Phi_{1}(b / Y)$ is a bend curve which precisely can be approximated by the parabola: $\Phi_{1}(b / Y)=4+b / Y+4(b / Y)^{2}$, or by a power form:

$\Phi_{1}(b / Y)=4+5(b / Y)^{1.8}$

Thus $M_{y}=f_{c} \frac{b h^{2}}{6}\left(1-\frac{N_{u}}{N_{u, m}}\right)\left[3-\frac{1-N_{u} / N_{u, m}}{s+1} \cdot\left(4+5\left(\frac{b}{Y}\right)^{1.8}\right)\right]$

and according to Eq.(13) and (16) is:

$\frac{M_{y}}{M_{y, \infty}}=1-\frac{\left(1-N_{u} / N_{u, m}\right) \cdot 5(b / Y)^{1.8}}{3 s-1+4 N_{u} / N_{u, m}}$

and $b / Y$ is directly known from: $M_{y}, M_{y, \infty}$ and $N$, where $M_{y}$ is the component in the stiff direction of the ultimate biaxial moment and $M_{y, \infty}$ the direct calculable uniaxial strength in the stiff direction (thus $Y \rightarrow \infty$ or $b / Y=0$ in Eq. (56). For the component of the ultimate bi-axial bending strength in the weak direction $M_{z}$ is according to Eq. (15) in the range of $0 \leq b / Y \leq 1$ :

$$
M_{z}=f_{c} \frac{h b^{2}}{6} \cdot\left(1-\frac{N_{u}}{N_{u, m}}\right) \cdot \Phi_{2}(b / Y)=f_{c} \frac{h b^{2}}{6} \cdot\left(1-\frac{N_{u}}{N_{u, m}}\right) \cdot\left(\frac{b}{Y}+0.5\left(\frac{b}{Y}\right)^{1.8}\right)
$$

because

$$
\Phi_{2}(b / Y)=\frac{1-b / 2 Y}{-1+Y / b+b / 3 Y} \approx b / Y+0.644(b / Y)^{2}-0.144(b / Y)^{3} .
$$

or by a power form:

$$
\Phi_{2}(b / Y)=b / Y+0.5(b / Y)^{1.8}
$$

According to Eq. (22) is:
$\frac{V_{u}}{V_{0, \infty}}=\left(1-\frac{N_{u}}{N_{u, m}}\right) \Phi_{3}(b / Y)$ with:

$\Phi_{3}(b / Y)=\frac{1-b / 2 Y}{1-b / Y+b^{2} /\left(3 Y^{2}\right)}=\frac{\Phi_{2}(b / Y)}{b / Y}$.

Thus:

$\frac{V_{u}}{V_{0, \infty}}=\left(1-\frac{N_{u}}{N_{u, m}}\right) \cdot\left(1+0.5\left(\frac{b}{Y}\right)^{0.8}\right)$.

\subsection{Dominating Bending in the Weak Direction Stiff}

The equations of Section 3.2 apply with interchange of $b$ with $h$ and $Y$ with $Z$ and $y$ with $z$

Thus for dominating bending in the weak direction when $Y \leq b$ and $Z \geq h$ :

$$
\begin{aligned}
& M_{z}=f_{c} \frac{h b^{2}}{6}\left(1-\frac{N_{u}}{N_{u, m}}\right)\left[3-\frac{1-N_{u} / N_{u, m}}{s+1} \cdot\left(4+5\left(\frac{h}{Z}\right)^{1.8}\right)\right], \\
& \frac{M_{z}}{M_{z, \infty}}=1-\frac{\left(1-N_{u} / N_{u, m}\right) \cdot 5(h / Z)^{1.8}}{3 s-1+4 N_{u} / N_{u, m}}, \\
& M_{y}=f_{c} \frac{b h^{2}}{6} \cdot\left(1-\frac{N_{u}}{N_{u, m}}\right) \cdot\left(\frac{h}{Z}+0.5\left(\frac{h}{Z}\right)^{1.8}\right), \\
& \frac{V_{u}}{V_{0, \infty}}=\left(1-\frac{N_{u}}{N_{u, m}}\right) \cdot\left(1+0.5\left(\frac{h}{Z}\right)^{0.8}\right) .
\end{aligned}
$$

\subsection{Dominating Tension}

The foregoing equations also apply for tension with bending and will cover the cases in practice. For dominating tension, the calculation according to Section 2.4.1 to 2.4.3 of Fig. 7 should be followed. These equations can not be simplified and should be tabulated for different values of $h / Z$ and $b / Y$ or solved by a numerical method for a given loading. Because for high tension and for lower qualities and large structural sizes the (long term) tensile strength will be lower than the compression strength and the behaviour is linear elastic, based on the ultimate tensile stress according to Section 2.4.4:

$1-N_{u} / N_{u}=M_{y} / M_{u, y}+M_{z} / M_{u, z}$.

\section{DESIGN EQUATIONS}

The given equations for biaxial bending are easy programmable for numerical solutions. However it always is necessary to provide simple Code rules.

The boundary conditions of application of the equations are determined by the uniaxial bending cases and therefore the conditions:

$1 \geq N_{u} / N_{u, m} \geq-(s-1) / 2$

$V_{0, \infty}=\frac{2}{3} f_{v} b h \frac{2}{s+1}=\frac{2}{3} f_{v, f} b h$.

$V_{u, \infty} / V_{0, \infty}=1-N_{u} / N_{u, m}$. 
apply for dominated bending in the stiff and weak direction and for dominated tension according to Sections 2.1, 2.2 and 2.4.

For dominating high compression, and therefore low shear loading and by $N$ reduced bending moments according to Eqs.(32) and (33) of Section 2.3, applies:

$((5-s) / 6) \leq N_{u} / N_{u, m} \leq 1$,

$V_{u}=2 f_{v} b h\left(1-N_{u} / N_{u, m}\right) /(s+1)=f_{v, f} b h \leq f_{v} b h / 3$,

$M_{y} \leq f_{c} b h^{2}\left(1-N_{u} / N_{u, m}\right) / 4 \leq f_{c} b h^{2}(1+s) / 24$,

$M_{z} \leq f_{c} h b^{2}\left(1-N_{u} / N_{u, m}\right) / 4 \leq f_{c} h b^{2}(1+s) / 24$.

The boundary conditions for application of the other bending moment equations are for:

Dominating bending in the stiff direction, Section 2.1:

$f_{c} b h^{2}(s+1) / 24 \leq M_{y} \leq f_{c} b h^{2}(s+1) / 12$ and

$0 \leq M_{z} \leq f_{c} b h^{2}(s+1) / 24$.

Dominating bending in the weak direction, Section 2.2:

$0 \leq M_{y} \leq f_{c} h b^{2}(s+1) / 24$ and

$f_{c} h b^{2}(s+1) / 24 \leq M_{z} \leq f_{c} h b^{2}(s+1) / 12$.

The same conditions apply for $M_{y}$ and $M_{z}$ of dominating tension according to Sections 2.4.1 to 2.4.3. However as Code rule, these equations are too extended and should be replaced for design for dominating tension by the save Eq.(53).

Thus for dominating compression, when $Z \leq h$ and $Y \leq b, \operatorname{Eqs}(28)$ to (34) apply. Else design can be based on the equations of Section 3.

Eq.(54) can be written, using Eq.(18):

$\frac{M_{y}}{f_{m} b h^{2} / 6}=\left(1-\frac{N}{N_{u, m}}\right)\left[\frac{(3 s+3)-\left(1-N / N_{u, m}\right) \Phi_{1}(b / Y)}{3 s-1}\right]$,

which will be written:

$m_{y}=(1-n)\left(\frac{(3 s+3)-(1-n) \Phi_{1}}{3 s-1}\right)=\frac{3 s+3}{3 s-1}(1-n)-(1-n)^{2} \frac{\Phi_{1}}{3 s-1}$.

Similarly Eq.(58) can be written:

$m_{z}=(1-n) \cdot \frac{s+1}{3 s-1} \Phi_{2}$.

This is a linear relation between $m$ and $n$, the same as applies for the shear strength Eq. (24).

According to the 3-point bending test is: $V_{u} a=M_{u}$, where $a=L / 2$ is the distance of the load in the middle of the beam to the support. Thus $a / h=M_{u} / V_{u} h$ is the shearslenderness with critical value:

$a_{c} / h=\left(f_{m} b h^{2} / 6\right) /\left((2 / 3) f_{v, f} b h^{2}\right)=f_{m} / 4 f_{v, f}$.

This critical value of maximal bending and maximal shear failure at the same time is $a_{c} / h \approx 3$ according to the test-beam dimensions of the shear strength test for mean quality European softwoods. Eq. (24) thus may be written:

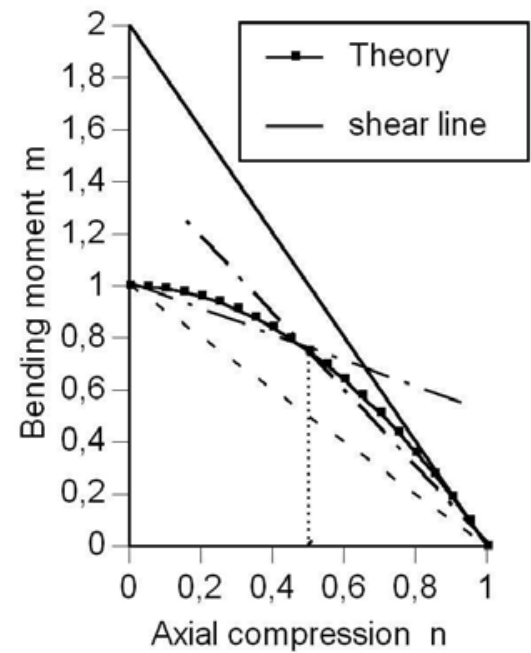

Fig. (8). Interaction curve cut off (by the dashed bending- compression strength shear line or no cut off by the drawn ultimate shear line).

$\frac{V_{u, \infty}}{V_{0, \infty}}=\frac{M_{y} a_{c}}{M_{y, \infty} a}=\frac{3 h M_{y}}{M_{y, \infty} a}=1-\frac{N}{N_{u, m}}$ or:

$n=1-(3 h / a) m_{y}$

For $a=3 h$, the boundary is reached where below the maximal possible bending moment will be reduced by the maximal possible shear force and Eq.(68) then becomes:

$n=1-m_{y}$.

This linear relation was the basis of the Dutch Code [7], and should apply for all Codes as long as the shear calculation according to Eq. (68) is absent. Eq. (68) shows the parabolic Eurocode line to be a factor 2 too unsafe when $a=3 h$ (see Fig. (8) and [3]).

Because such a linear relation also is determining for one component of the biaxial strength as given by Eq. (67). The curved line Eq, (66) the best also can be approximated by 2 straight lines through the end points $(m=1 ; \mathrm{n}=0)$ and $(m=$ $0 ; n=1)$, and the point on the curve for $n=0.5$ (see [3]) where according to Eq. (66):

$m_{y}=0.5 \frac{3 s+3-\Phi_{1} / 2}{3 s-1}$,

for $n=0.5$, giving:

$m_{y}=1-\frac{3 s-5+\Phi_{1} / 2}{3 s-1} n \quad$ when $n \leq 0.5$,

$n=1-\frac{3 s-1}{3 s+3-\Phi_{1} / 2} m_{y}$ when $n \geq 0.5$.

The same equations apply for dominating bending in the weak direction after interchange of $b$ with $h$ and $Y$ with $Z$ and $y$ with $z$.

\section{CONCLUSIONS}

A derivation is given of the biaxial bending strength in accordance with the limit analysis method and thus based on elastic-full-plastic behaviour. Therefore, with the restriction of neglecting hardening stages after initial "flow", the analy- 
sis is rigorous and the strength prediction realistic and the result has to be applied in the Building Codes to provide the prescribed sufficient precise reliability calculation.

For the highest lower bound solution of biaxial bending strength is necessary that the neutral axis is a straight line and that unlimited flow in pure compression occurs, thus when there is bending-tension failure and when the shear stress is carried in the elastic part of the cross section. This is an improvement with respect to the thus far applied old model [6] restricting the ultimate plastic compression strain.

The derived general expressions in coordinates of the boundary line of the full compression area are simplified to elementary design equations, which also has to be in the Regulations. Chosen is, for simplicity of design, for separate ultimate shear strength and ultimate bending-compression strength equations.

The equations contain also the solution for uniaxial bending cases, which are already shown to precisely explain and fit data by the elastic full plastic limit approach.

The value of $s=f_{t} / f_{c}$ appears to be about constant for all load combinations of bending with compression, indicating again that there always is failure by the ultimate tensile strength. A volume effect by stress distribution thus needs not to be regarded as follows from the uniaxial data. The volume effect thus now is caused by the volume alone due to decreasing quality by volume increase.

\section{CONFLICT OF INTEREST}

The authors confirm that this article content has no conflicts of interest.

\section{ACKNOWLEDGEMENT}

Declared none.

\section{REFERENCES}

[1] van der Put TACM. A continuum failure criterion applicable to wood. J Wood Sci 2009; 55: 315-22.

[2] Design of Timber Structures, Part 1-1: General - Common rules and rules for buildings Eurcode 5, EN 1995: 2004

[3] van der Put TACM. Failure criterion for timber beams loaded in bending, compression and shear. Wood Mat Sci Eng 2010; 1: 41-9

[4] Chen WF, Atsuta T. Theory of beam columns, Space behavior and design. USA: J. Ross Publishing 2007, vol. 2.

[5] Kollmann F. Technologie des Holzes und der Holzwerkstoffe. Berlin: Springer Verlag 1951.

[6] Johns KC, Buchanan AH. Strength of timber members in combined bending and axial loading. CIB/IUFRO Meeting Boras, Sweden 1982. (see also CIB W18, 1985 Israel).

[7] Timber structures - Basic requirements and determination Methods. NEN 6760 - TGB 1990.

Received: July 07, 2012

(C) T. A. C. M. Van Der Put; Licensee Bentham Open.

This is an open access article licensed under the terms of the Creative Commons Attribution Non-Commercial License (http://creativecommons.org/licenses/by-nc/3.0/) which permits unrestricted, non-commercial use, distribution and reproduction in any medium, provided the work is properly cited. 